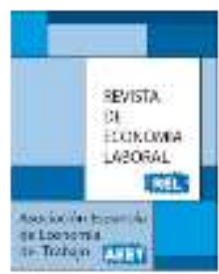

\title{
EMPLEO Y FORMALIZACIÓN LABORAL JUVENIL EN COLOMBIA. UNA EVALUACIÓN DE LA LEY DEL PRIMER EMPLEO
}

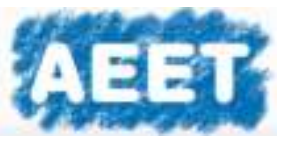

\author{
John Ariza ${ }^{1}$ y Paola Cedano \\ Universidad de Tolima
}

Recibido Noviembre 2017; Aceptado Marzo 2018

\section{Resumen}

En el presente documento se analiza el impacto de la ley 1429 de 2010 sobre el empleo y la formalización laboral de los jóvenes en Colombia. La evaluación de impacto se realiza a partir de un modelo de diferencias en diferencias considerando los primeros y últimos trimestres de los años anteriores y posteriores a la entrada en vigencia la ley. Los resultados indican que los efectos de la ley fueron diferenciales sobre grupos sociodemográficos de jóvenes tanto en el corto como en el largo plazo. Aunque en el corto plazo la ley aumentó la probabilidad de encontrar empleo para este grupo en general, también aumentó la probabilidad de encontrarse en situación de informalidad. Casi un año después de la aplicación de la ley, la probabilidad de estar ocupado disminuyó al igual que la probabilidad de ser informal. Los jóvenes más cualificados sin embargo, afrontaron menores probabilidades de empleo en el trimestre posterior a la reforma y mayores niveles de informalidad después de varios meses de la implementación de la ley.

Palabras clave: empleo, informalidad laboral, política pública.

Clasificación JEL: J21, J46, J48.

1 Departamento de Economía y Finanzas, Universidad del Tolima, Ibagué-Colombia. E-mail: jfariza@ut.edu.co.

(C) Revista de Economía Laboral 


\title{
Employment and youth labour formalization in Colombia. An assessment of the law of first employment
}

\begin{abstract}
In this paper, we study the impact of the law 1429 of December 2010 on both employment and labor formalization of young workers in Colombia. To asset the policy impact, we use an econometric model of differences in differences considering the first and the last quarter of the periods before and after the law was applied. Results suggest different effects of the law on young socio-demographic groups both in the short and in the long run. In the short run, the law increased the probability of finding a job but also the probability of being an informal worker. Several months later, both the probability of finding a job and the probability of being informal decreased. The most educated young workers, however, faced lower probabilities of employment in the quarter after the law and higher levels of informality one year later.
\end{abstract}

Key words: employment, labour informality, public policy.

JEL Classification: J21, J46, J48. 


\section{Introducción}

La inserción exitosa de los jóvenes en el mercado de trabajo constituye un tema de gran interés en la agenda actual de los gobiernos locales y nacionales. De acuerdo con la Organización Internacional del Trabajo-ILO (2016), el desempleo, el subempleo y la informalidad laboral golpea con más fuerza a la población joven que al resto de la población activa. El informe estima en 2016 un total de 71 millones de jóvenes desempleados en el mundo y un total 53 millones de jóvenes ocupados en países en vías de desarrollo que viven en situación de pobreza extrema o moderada. De acuerdo con indicadores laborales de la Gran Encuesta Integrada de Hogares GEIH realizada por el Departamento Administrativo Nacional de estadística-DANE (2015), los jóvenes entre 18 y 29 años de las trece ciudades y áreas metropolitanas en Colombia aportaron en el segundo trimestre del 2015 el 50\% del desempleo, el 26\% del subempleo y el $21 \%$ de la informalidad laboral total. La tasa de desempleo se calcula sobre la población activa y la tasa de subempleo e informalidad sobre la población ocupada.

Una de las políticas más importantes en los últimos años dirigida a mejorar las condiciones de inserción y calidad del empleo para los jóvenes en Colombia fue la Ley 1429 del 2010. Esta ley buscaba la generación y formalización del empleo a través de estímulos fiscales para empresas en sus etapas iniciales y para la contratación de grupos poblacionales en desventaja. En particular, la ley contempló incentivos para los jóvenes menores de 28 años tales como la creación de programas de microcrédito y crédito para empresas tanto del sector rural como el urbano, la reducción sustantiva en los trámites y una mayor asistencia técnica. La ley estableció beneficios en exenciones tributarias para las empresas que contratasen jóvenes tales como progresividad en el pago de la matrícula mercantil, en el impuesto a la renta y en los parafiscales. Los aportes parafiscales son contribuciones obligatorias sobre la nómina que financian: El Instituto Colombiano de Bienestar Familiar (ICBF), El Servicio Nacional de Aprendizaje (SENA) y las Cajas de Compensación Familiar.

El objetivo del presente artículo es evaluar precisamente el impacto que tuvo la implementación de la ley 1429 de 2010 sobre el empleo y la formalización laboral de los jóvenes entre 18 y 28 años de edad en Colombia durante el periodo 2010 y 2011. A pesar de la relevancia del tema, en la actualidad existen pocos estudios que aborden la evaluación cuantitativa de dicha ley. El análisis se realiza sólo durante este periodo debido a que en el año 2012 se aprobó una reforma tributaria 
que modificó los incentivos iniciales y que no permite identificar por tanto el efecto de la ley en años posteriores. Algunas preguntas que guían el presente artículo son: ¿Mejoraron los indicadores de contratación y calidad de empleo juvenil en el periodo inmediatamente posterior a la implementación de la ley? ¿Tuvo la ley efectos diferenciales sobre los indicadores laborales de hombres y mujeres?

La evaluación de la ley se realiza mediante un modelo de diferencias en diferencias en el que el grupo de control son las cohortes de población mayores de 28 años que no fueron afectadas por la reforma y el grupo de tratamiento son las cohortes de población juvenil afectadas por la reforma. Dado que la ley no sólo afectó a los jóvenes sino también a determinados grupos sociodemográficos desfavorecidos, para identificar el efecto de la reforma sólo sobre los jóvenes, se excluye de la muestra la población que cumple con otras características de vulnerabilidad tales como ser madres cabeza de familia y ser mujeres mayores de 40 años. El efecto de la reforma se estudia sobre la probabilidad de tener empleo y sobre la probabilidad de tener un empleo informal utilizando un modelo logit. El indicador de informalidad en este caso es el propuesto por el DANE que combina información sobre posición ocupacional, nivel educativo y tamaño de empresa del trabajador.

El artículo se divide en seis secciones además de esta introducción. En la segunda sección se presenta una descripción de la ley del primer empleo. En la tercera sección se hace una revisión de los principales estudios realizados en Colombia sobre esta reforma y sobre reformas similares. En la cuarta sección se explica la estrategia empírica. En la quinta sección se describen los datos y se describen algunos hechos estilizados del mercado laboral juvenil. En la sexta sección se presentan los resultados econométricos de la evaluación y se discuten las implicaciones. Finalmente, en la séptima sección se concluye.

\section{Descripción de la Ley de primer empleo}

La ley 1429 de 2010 tuvo por objeto mejorar los indicadores de empleo y formalización laboral de las pequeñas empresas y grupos poblacionales en condiciones de desventaja como jóvenes menores de 28 años, mujeres mayores de 40 y personas en situación de desplazamiento, en proceso de reintegración o en condición de discapacidad. Desde el punto de vista de la formalización empresarial, la ley creó incentivos mediante programas de desarrollo empresarial, progresividad en el pago de impuesto sobre la renta, de impuestos a la nómina, de impuesto de 
industria y comercio y de matrícula mercantil a pequeñas empresas en sus etapas iniciales.

Con relación a los incentivos para la generación de empleo y formalización laboral de grupos vulnerables, la ley generó descuentos en el impuesto sobre la renta y complementarios de los aportes parafiscales y otras contribuciones de nómina a quienes contratasen personas en estas condiciones o a trabajadores de bajos ingresos. La ley simplificó además los trámites laborales y comerciales para facilitar la formalización.

Para el presente estudio, los indicadores laborales de los jóvenes en esta ley se afectan por dos vías. En primer lugar, en el capítulo de formalización empresarial, los incentivos para el desarrollo empresarial generaron nuevos programas de crédito y microcrédito para empresas creadas por jóvenes menores de 28 años mediante herramientas financieras como incentivos a las tasas de interés, al capital, periodos de gracia y programas de formación, capacitación, apoyo técnico, financiero y asesoría especializada.

En segundo lugar, los incentivos fiscales que creó la ley a las empresas estaban condicionados a la contratación de nuevos trabajadores pertenecientes a los grupos poblaciones comentados previamente entre los cuales se encuentran los jóvenes menores de 28 años. En este caso, las empresas toman los aportes al SENA, ICBF, Cajas de Compensación Familiar, Fondo de Seguridad y Garantía en Salud Fosyga y Fondo de Garantía de Pensión Mínima como descuento tributario para la determinación del impuesto de renta $\mathrm{y}$ complementarios. Los beneficios en este caso sólo tienen un periodo de dos años por empleado.

Hay que destacar que los beneficios establecidos por la ley de primer empleo se modificaron al entrar en vigencia la ley 1607 de diciembre de 2012 en la que se crea el Impuesto sobre la Renta para la Equidad-CREE. Con la entrada del nuevo impuesto se exonera del pago de los aportes por parafiscales y al sistema de seguridad social en salud a los empleados que devenguen menos de diez salarios mínimos. Son deducibles además del impuesto a la renta y complementarios, las contribuciones que efectúan las entidades empleadoras a los fondos de pensiones de jubilación, invalidez y cesantías. De este modo, los beneficios por emplear a personas de grupos vulnerables o de bajos ingresos se anulan, debido a que el beneficio sólo se encontraría en la exoneración de los aportes a cajas de compensación familiar. 


\section{Antecedentes}

El desarrollo de la literatura sobre evaluación de impacto de políticas laborales en Colombia inicia con la implementación de la Ley 789 de 2002. Esta ley buscaba flexibilizar el mercado laboral a través de tres aspectos: la ampliación de la jornada laboral, la reducción de los costos de despido sin justa causa e indemnización y la modificación a los contratos de aprendizaje. El marco de dicha ley suponía que la flexibilización laboral generaría un mayor número de empleos en Colombia. Sin embargo, la literatura señala que el efecto general de la reforma no fue tan grande como se esperaba y que tuvo efectos diferenciales positivos y negativos sobre grupos de trabajadores y regiones. Para una revisión profunda los alcance de esta ley a nivel nacional ver Isaza (2003), Cárdenas y Barrera (2003), Castaño, López y Rhenals (2004), Gaviria (2004), Núñez (2005), Amarante y Santamaría (2005) y Guataqui y García (2009). El impacto de la ley a nivel regional se estudia en López (2007), Polanco, Cerón y Franco (2015) y Narváez (2013).

La literatura que evalúa el efecto de la ley 1429 de 2010 sobre los indicadores del mercado laboral colombiano es en general escasa. Existen pocos estudios que realicen una evaluación de impacto de esta política de empleo y formalización laboral para los grupos hacia los cuales estaba dirigida. Farné (2011) analiza el efecto de esta ley utilizando estadísticas descriptivas del mercado de trabajo. El estudio concluye que no hay incentivos contundentes en materia de empleo y que la mayor probabilidad de encontrar trabajo se debe primordialmente al buen momento del mercado de trabajo nacional. Así mismo, estudios realizados por Farné (2012) y Jaramillo (2016), constatan el efecto tímido de la ley sobre el empleo juvenil.

En 2013, Econométrica-SEI (Sistemas Especializados de Información S.A.) realizan un estudio para el Ministerio de Trabajo, en el cual analizan el impacto de la ley sobre los pequeños empresarios y sobre los grupos poblacionales vulnerables utilizando modelos de diferencias en diferencias estimados mediante la técnica de Propensity Score Matching. Los datos para el grupo tratamiento se obtuvieron de una selección aleatoria de pequeños empresarios en el RUES (Registro Único Empresarial y Social de las Cámaras de Comercio) mientras que para el grupo control se realizó un operativo telefónico. Los resultados sugieren logros en formalización empresarial, pero efectos tímidos en la formalización laboral y fiscal. De acuerdo con la información en Plantilla Integrada de Liquidación de Aportes -PILA para 2012, sólo el 1,5\% de las 
empresas potencialmente beneficiarias para aplicar el descuento por contratación de grupos poblacionales, lo hicieron.

La evaluación para uno de los grupos específicos para los que estaba dirigida la ley la realiza Rodríguez (2013). En su estudio estima el impacto de la ley sobre indicadores de empleo de las mujeres mayores de 40 años durante el 2011-2012 utilizando un modelo diferencias en diferencias y un modelo de regresión discontinua como prueba de robustez. Los resultados por las dos metodologías sugieren que el ingreso laboral no aumentó y que la probabilidad de estar empleadas disminuyó para los años 2011 y 2012. Con relación al desempleo, sugiere que la probabilidad de estar en esta condición sólo disminuyó un 1,5\% entre 2010 y 2011. El estudio concluye por tanto que los efectos de la política han sido pequeños para este grupo poblacional.

Con relación al efecto de la ley a nivel de ciudades se pueden relacionar dos estudios. En el primero, Alturo (2015) analiza el impacto de la ley para el Espinal (Tolima) realizando un análisis descriptivo y exploratorio por medio de una encuesta a representantes legales y contadores públicos de las empresas formalizadas con el fin de analizar el impacto tributario generado por la ley en la formalización de la pequeña empresa. De acuerdo con sus resultados, el efecto de la ley fue pequeño ya que de las nuevas pequeñas empresas creadas durante ese periodo sólo siete se acogieron al beneficio de la ley y sólo 18 de las creadas renovaron su matrícula mercantil el siguiente año.

Espinel (2016), evalúa la implementación de la ley para Bogotá considerando los incentivos otorgados a través de aportes parafiscales a las pequeñas empresas. En su estudio evalúa los resultados obtenidos a través del tiempo y el costo fiscal generado. De acuerdo con sus resultados, la ley no permitió solucionar el problema de la informalidad en las micro y pequeñas empresas. El estudio cuestiona la política de generación de empleo y formalización laboral enfocada únicamente en disminución de cargas salariales, prestacionales, tributarias o de seguridad social para las empresas en la medida en que no se alcanzan los objetivos de ley pero sí se mejora la situación económica de las empresas.

De acuerdo con lo anterior, es poca la literatura que estudia el impacto cuantitativo de la ley sobre los indicadores de empleo e informalidad en los jóvenes en Colombia. Aunque los estudios coinciden en efectos pequeños para los diferentes grupos poblacionales cobijados por la ley, no hay una medida específica relacionada con el impacto sobre la probabilidad de empleo e informalidad juvenil una vez entra en vigencia esta ley. Este artículo tiene como objetivo precisamente 
contribuir a la literatura sobre el efecto de la ley estimando el impacto sobre estos indicadores laborales juveniles considerando género y nivel educativo.

\section{Metodología}

En el presente artículo se considera el método de diferencias en diferencias. El método de diferencias en diferencias es una técnica econométrica ampliamente utilizada en la evaluación de impacto de las políticas públicas que consiste en estimar el efecto causal de la política (X) sobre una variable resultado (Y) en el contexto de experimentos naturales o cuasi experimentos (Card, 1990; Gaviria, 2005; Bernal y Peña, 2014). Esta técnica difiere del método de evaluación de impacto de diferencias en la medida en que los datos no provienen propiamente de un experimento aleatorio diseñado para este propósito (experimento aleatorio controlado) sino que han sido recolectados para otros fines en un periodo en el cual se presentó un shock exógeno o se implementó una política.

Debido a que la información en este estudio proviene de encuestas de hogares en periodos previos y posteriores a la implementación de la ley, en el presente documento se utilizará un modelo de diferencias en diferencias para datos de corte transversal repetidos. De acuerdo con Bernal y Peña (2014) aunque en este caso, los individuos que conforman la muestra en cada periodo son diferentes, el supuesto de que las muestras en ambos periodos son aleatorias de la misma población, implica que los individuos en el periodo previo a la reforma pueden ser utilizados como sustitutos de los individuos de los grupos tratamiento y control del corte transversal posterior.

Para implementar el estimador de diferencias en diferencias en datos de corte transversal repetido se requiere que sea posible identificar a los individuos del grupo tratamiento y que la composición en términos de variables no observables de quienes van a estar en el grupo tratamiento y el grupo control permanezca constante (Bernal y Peña, 2014, p80). Para este caso, el grupo tratamiento está plenamente identificado: jóvenes entre 18 y 28 años de edad que viven en las principales ciudades y áreas metropolitanas del país y que no son madres cabeza de familia. El grupo control serán aquellas personas mayores de 28 años que además de las características anteriores, no sean mujeres mayores de 40 años.

Aunque la ley también afectó a las personas discapacitadas y a las personas en situación de desplazamiento, no se consideran en la 
definición de los grupos debido a que no es posible identificarlas en las encuestas de hogares. Con relación a la composición de los grupos en términos de variables no observables, suponemos que estas son similares en la muestra considerada y no se afectan por la implementación de la política con la definición adoptada.

De acuerdo con Wooldridge (2013), el modelo de regresión para obtener el efecto de la política en el contexto de los experimentos naturales se presenta en la ecuación (1):

$$
Y_{i}=\beta_{0}+\beta_{1} D_{i}+\beta_{2} T_{i}+\beta_{3} D_{i} T_{i}+u_{i}
$$

Donde $\mathrm{Y}_{\mathrm{i}}$ representa la variable resultado, $\mathrm{D}_{\mathrm{i}}$ es la dummy que indica si el individuo pertenece o no al grupo tratamiento (afectado por la ley), Ti es la dummy de tiempo que indica si la observación es previa o posterior a la reforma, $\mathrm{D}_{\mathrm{i}} \mathrm{T}_{\mathrm{i}}$ es la interacción entre el indicador de tratamiento y la dummy de periodo de implementación de la política, y $\mathrm{u}_{\mathrm{i}}$ es el término de perturbación. En este marco, el coeficiente asociado a la variable interacción $\left(\beta_{3}\right)$ es el efecto causal asociado a la política obtenido por el método de diferencias en diferencias. En términos de esperanzas matemáticas condicionales, este efecto viene dado por la siguiente expresión:

$$
\begin{aligned}
& {[E(Y \mid D=1, T=1)-E(Y \mid D=1, T=0)]-[E(Y \mid D=0, T=1)-} \\
& E(Y \mid D=0, T=0)] \quad(2)
\end{aligned}
$$

Al tomar las medias muestrales de los grupos de tratamiento y control antes y después de la reforma, encontramos que la ecuación (2) se puede expresar como:

$$
\hat{\tau}_{\text {dif-en-dif }}=(\Delta \bar{Y} \mid D=1)-(\Delta \bar{Y} \mid D=0)
$$

En el que se observa la diferencia en medias de la variable dependiente de los individuos tratados antes y después de la implementación de la política respecto de la diferencia en medias de los individuos en el grupo control antes y después de la implementación de la política es el estimador del efecto de la política $\hat{\tau}$ en el modelo de diferencias en diferencias (ecuación 3). En términos del modelo de regresión (ecuación 1) y de acuerdo con la ecuación (2), este estimador $\hat{\tau}$ se traduce en: 


$$
\begin{gathered}
\hat{\tau}_{\text {dif-en-dif }}=\left[\left(\hat{\beta}_{0}+\hat{\beta}_{1}+\hat{\beta}_{2}+\hat{\beta}_{3}\right)-\left(\hat{\beta}_{0}+\hat{\beta}_{1}\right)\right]-\left[\left(\hat{\beta}_{0}+\hat{\beta}_{2}\right)-\left(\hat{\beta}_{0}\right)\right] \\
\tau_{\text {dif-en-dif }}=\hat{\beta}_{3}
\end{gathered}
$$

Que es precisamente el efecto del impacto de la política. Para el caso de la Ley 1429 es fácil ver en las ecuaciones (5) y (6) que el efecto de la política sobre la ocupación o la informalidad en la población juvenil está asociado al coeficiente de la variable interacción Joven $i * T 2011_{i}$. Estas ecuaciones difieren de la ecuación (1) en la medida en que incluyen un vector $\mathrm{X}_{\mathrm{i}}$ de $\mathrm{k}$ características observables de los individuos relacionadas con el sexo y el nivel educativo y un vector $Z_{i}$ de $m$ interacciones entre joven, tiempo y sexo y joven, tiempo y nivel educativo.

$$
\begin{gathered}
\text { Ocupado }_{i}=\beta_{0}+\beta_{1} \text { Joven }_{i}+\beta_{2} \text { T2011 }_{i}+\beta_{3} \text { Joven }_{i} \text { T2011 }_{i_{i}}+\gamma_{k} X_{i} \\
\\
+\delta_{m} Z_{i}+u_{i}
\end{gathered}
$$

Esta extensión se realiza para evaluar el efecto de la Ley en diferentes grupos sociodemográficos de jóvenes. Debido a que las variables dependientes ocupación e informalidad en este sistema de ecuaciones son dicótomas, el efecto de la reforma se expresa en términos de probabilidad de estar ocupado o de ser informal. En este contexto, la estimación de estas ecuaciones se realizará utilizando un modelo logit que considera la naturaleza cualitativa de la variable dependiente.

\section{Datos y descriptivos}

El presente artículo utiliza datos de corte transversal para el primer y cuarto trimestre del 2010 y del 2011 provenientes de la Gran Encuesta Integrada de Hogares (GEIH). La información proveniente del Departamento Administrativo Nacional de Estadística (DANE) se procesó de acuerdo con los lineamientos establecidos por la entidad. La muestra está conformada sólo por las 13 ciudades y áreas metropolitanas de Colombia. En el procesamiento de los datos se crearon nuevas variables necesarias para la estimación como la informalidad laboral y se excluyeron las observaciones que no reportaban datos en las variables consideradas. La medida de informalidad laboral considerada en este artículo es la establecida por el DANE en la cual un ocupado es informal 
si cumple alguna de las siguientes condiciones: es un asalariado que trabaja en una empresa privada que ocupa hasta 5 personas; es un trabajador familiar sin remuneración; es un empleado doméstico o jornalero o peón o patrón o trabajador cuenta propia en una empresa hasta de cinco trabajadores.

En el cuadro 1 se presentan algunos indicadores del mercado laboral colombiano para las trece principales ciudades y áreas metropolitanas del país. En el panel superior se muestra el total de población y las tasas globales de participación, ocupación, desempleo e informalidad durante el periodo de estudio por trimestres con sus respectivas variaciones. En el panel inferior se presenta información sobre la población joven en el grupo tratamiento, las tasas de ocupación desempleo e informalidad y la participación en puntos porcentuales de este grupo en las tasas generales.

De acuerdo con la información del cuadro, tanto la participación laboral como la tasa de ocupación para las principales áreas urbanas del país aumentaron, mientras que la tasa de desempleo y la tasa de informalidad cayeron tanto en el primer trimestre como en el último. Las caídas en el desempleo y la informalidad en esos periodos son consistentes con la tendencia nacional. Durante el 2009-2012 la tasa de desempleo en las áreas urbanas pasó de 14\% al 10\% mientras que la tasa de informalidad pasó del 52\% en 2009 a 51.2\% en 2012. Es conveniente señalar que en los últimos trimestres de cada año la ocupación crece a una mayor velocidad que la reducción en la informalidad.

Los indicadores laborales de los jóvenes del grupo tratamiento en el mercado de trabajo urbano se presentan en el panel inferior del cuadro 1. De acuerdo con estos datos, este grupo es fuertemente golpeado por el desempleo con tasas de más de siete puntos porcentuales por encima del promedio de las 13 ciudades. En términos de participación porcentual en el total de la tasa, los jóvenes en el grupo tratamiento aportan un poco más de 3 puntos porcentuales en el caso del desempleo y al menos 7 puntos porcentuales en el caso de la informalidad. La variación de los indicadores de participación, ocupación e incluso informalidad está en línea con la tendencia a nivel agregado. 
Cuadro 1: Indicadores del mercado laboral. Trece ciudades y áreas metropolitanas.

\begin{tabular}{|c|c|c|c|c|c|c|}
\hline \multicolumn{7}{|c|}{ Total población } \\
\hline & 2010 & 2011 & Variación & 2010 & 2011 & Variación \\
\hline & $\begin{array}{l}\text { Ene- } \\
\text { Mar }\end{array}$ & $\begin{array}{l}\text { Ene- } \\
\text { Mar }\end{array}$ & & $\begin{array}{l}\text { Oct- } \\
\text { Dic }\end{array}$ & $\begin{array}{l}\text { Oct- } \\
\text { Dic }\end{array}$ & \\
\hline $\begin{array}{l}\text { Población } \\
\text { (miles) }\end{array}$ & 19.861 & 20.127 & & 20.060 & 20.327 & \\
\hline $\begin{array}{l}\text { Activos } \\
\text { (miles) }\end{array}$ & 10.377 & 10.679 & & 10.747 & 11.187 & \\
\hline $\begin{array}{l}\text { Ocupados } \\
\text { (miles) }\end{array}$ & 8.955 & 9.251 & & 9.562 & 10.036 & \\
\hline $\begin{array}{l}\text { Desempleados } \\
\text { (miles) }\end{array}$ & 1.422 & 1.429 & & 1.186 & 1.151 & \\
\hline $\begin{array}{l}\text { Informales } \\
\text { (miles) }\end{array}$ & 4.577 & 4.679 & & 4.892 & 5.124 & \\
\hline $\begin{array}{l}\text { Tasa de } \\
\text { participación }\end{array}$ & 65,0 & 65,8 & 0,8 & 66,5 & 68,0 & 1,5 \\
\hline $\begin{array}{l}\text { Tasa de } \\
\text { ocupación }\end{array}$ & 56,1 & 57,0 & 0,9 & 59,1 & 61,0 & 1,9 \\
\hline $\begin{array}{l}\text { Tasa de } \\
\text { desempleo }\end{array}$ & 13,7 & 13,4 & $-0,3$ & 11,0 & 10,3 & $-0,7$ \\
\hline $\begin{array}{l}\text { Tasa de } \\
\text { informalidad }\end{array}$ & 51,6 & 50,9 & $-0,7$ & 51,7 & 51,3 & $-0,4$ \\
\hline \multicolumn{7}{|c|}{ Población joven en grupo tratamiento } \\
\hline $\begin{array}{l}\text { Población } \\
\text { (miles) }\end{array}$ & 2.765 & 2.803 & & 2.772 & 2.796 & \\
\hline $\begin{array}{l}\text { Ocupados } \\
\text { (miles) }\end{array}$ & 1.636 & 1.681 & & 1.752 & 1.805 & \\
\hline $\begin{array}{l}\text { Desempleados } \\
\text { (miles) }\end{array}$ & 439 & 444 & & 371 & 375 & \\
\hline $\begin{array}{l}\text { Informales } \\
\text { (miles) }\end{array}$ & 668 & 675 & & 704 & 700 & \\
\hline $\begin{array}{l}\text { Tasa de } \\
\text { ocupación }\end{array}$ & 59,0 & 60,0 & 0,9 & 63,2 & 64,6 & 1,4 \\
\hline $\begin{array}{l}\text { Tasa de } \\
\text { desempleo }\end{array}$ & 21,2 & 20,9 & $-0,3$ & 17,5 & 17,2 & $-0,3$ \\
\hline $\begin{array}{l}\text { Tasa de } \\
\text { informalidad }\end{array}$ & 40,9 & 40,2 & $-0,8$ & 40,2 & 38,8 & $-1,4$ \\
\hline \multicolumn{7}{|c|}{ Participación en puntos porcentuales en: } \\
\hline $\begin{array}{l}\text { Tasa de } \\
\text { ocupación }\end{array}$ & 10,2 & 10,4 & 0,1 & 10,8 & 11,0 & 0,1 \\
\hline $\begin{array}{l}\text { Tasa de } \\
\text { desempleo }\end{array}$ & 4,2 & 4,2 & $-0,1$ & 3,5 & 3,4 & $-0,1$ \\
\hline $\begin{array}{l}\text { Tasa de } \\
\text { informalidad }\end{array}$ & 7,5 & 7,3 & $-0,2$ & 7,4 & 7,0 & $-0,4$ \\
\hline
\end{tabular}

Fuente: cálculos propios con base en la GEIH. 
En el cuadro 2 se presentan indicadores de empleo e informalidad por características sociodemográficas y sectoriales del grupo tratamiento como sexo, nivel educativo y sector económico en el que se desempeñan. De acuerdo con el cuadro, la participación en el empleo total del grupo disminuyó durante el periodo, impulsado por una caída en el empleo femenino, una reducción en empleados con nivel educativo superior y una disminución en ocupados del sector comercio y otros servicios sociales. En este último caso, la perdida de participación en esos sectores se vio compensada en parte por una mayor participación en el sector hoteles restaurantes e industria manufacturera.

Cuadro 2: Población joven en el grupo de tratamiento.

\begin{tabular}{|c|c|c|c|c|c|c|}
\hline & \multicolumn{3}{|c|}{$\%$ en el empleo total } & \multicolumn{3}{|c|}{$\begin{array}{l}\text { \% de informalidad en el } \\
\text { empleo total }\end{array}$} \\
\hline & 2010 & 2011 & & 2010 & 2011 & \\
\hline & $\begin{array}{l}\text { Ene- } \\
\text { Mar }\end{array}$ & $\begin{array}{l}\text { Ene- } \\
\text { Mar }\end{array}$ & Variación & $\begin{array}{l}\text { Ene- } \\
\text { Mar }\end{array}$ & $\begin{array}{l}\text { Ene- } \\
\text { Mar }\end{array}$ & Variación \\
\hline Total & 18,23 & 18,18 & $-0,05$ & 7,46 & 7,30 & $-0,16$ \\
\hline \multicolumn{7}{|l|}{ Sexo } \\
\hline Hombre & 10,63 & 10,79 & 0,16 & 4,09 & 4,15 & 0,06 \\
\hline Mujer & 7,60 & 7,39 & $-0,21$ & 3,37 & 3,15 & $-0,22$ \\
\hline \multicolumn{7}{|l|}{$\begin{array}{l}\text { Nivel } \\
\text { educativo }\end{array}$} \\
\hline Primaria & 1,42 & 1,42 & 0,00 & 1,02 & 1,08 & 0,06 \\
\hline Secundaria & 9,20 & 9,26 & 0,06 & 4,70 & 4,70 & 0,00 \\
\hline Superior & 7,60 & 7,49 & $-0,11$ & 1,74 & 1,52 & $-0,22$ \\
\hline \multicolumn{7}{|c|}{ Sector económico } \\
\hline Agricultura & 0,15 & 0,15 & 0,00 & 0,10 & 0,11 & 0,01 \\
\hline Manufactura & 3,11 & 3,21 & 0,10 & 0,02 & 0,02 & 0,00 \\
\hline $\begin{array}{l}\text { Otras } \\
\text { industrias }\end{array}$ & 1,29 & 1,35 & 0,06 & 0,68 & 0,75 & 0,07 \\
\hline Comercio & 4,48 & 4,26 & $-0,21$ & 1,88 & 1,90 & 0,01 \\
\hline $\begin{array}{l}\text { Hoteles, } \\
\text { restaurantes }\end{array}$ & 2,78 & 3,10 & 0,33 & 1,40 & 1,48 & 0,08 \\
\hline $\begin{array}{l}\text { Educación y } \\
\text { salud }\end{array}$ & 1,63 & 1,69 & 0,06 & 1,42 & 1,49 & 0,07 \\
\hline Otros servicios & 4,79 & 4,39 & $-0,40$ & 3,21 & 2,87 & $-0,34$ \\
\hline
\end{tabular}

Fuente: cálculos propios con base en la GEIH.

Nota: los cálculos utilizan factores de expansión. 
En el caso de la informalidad, la participación del grupo disminuye y se debe fundamentalmente a una menor informalidad en las mujeres, en los más educados y en quienes trabajan en otros servicios comunales y sociales. El sector de hoteles y restaurantes registra aumentos en la participación en la informalidad total. De acuerdo con esto, es posible concluir que si bien es cierto la ocupación aumentó en el sector de hoteles y restaurantes, los jóvenes ocupados en esos sectores gozaron de una peor vinculación.

\section{Resultados econométricos}

En el cuadro 3 se presentan los resultados de la estimación del efecto de la ley 1429 sobre el empleo y la informalidad laboral de los jóvenes utilizando un modelo logit. Los resultados en cada caso se presentan bajo tres formas funcionales que indican el efecto de la reforma sobre diferentes grupos sociodemográficos definidos a partir de edad, niveles educativos y género. Los coeficientes de interés son los que muestran las interacciones entre la dummy de tiempo, la dummy joven y la dummy de educación secundaria, educación terciaria o género femenino según sea el caso. En este modelo, los coeficientes están expresados en términos de odds ratio en el marco del modelo logit.

De acuerdo con los resultados econométricos, la probabilidad de encontrar empleo en las principales ciudades y áreas metropolitanas en Colombia aumentó durante en el primer trimestre del 2011 respecto del mismo trimestre en el 2010 (columnas 1, 2 y 3). El logaritmo del ratio de probabilidades en la columna 3 indica que es 1,36 veces más probable que un joven esté empleado en 2011 que en 2010. Sin embargo, el efecto de la ley no fue homogéneo en este grupo. La probabilidad de empleo para las mujeres jóvenes disminuyó al igual que la probabilidad de empleo de jóvenes más cualificados. Este patrón de empleo por niveles educativos indicaría que el efecto de la reforma en el corto plazo incentivó la contratación en determinados sectores que utilizan en general mano de obra poco calificada. 


\section{Cuadro 3: Resultados (odds ratios) del modelo logit (primer trimestre).}

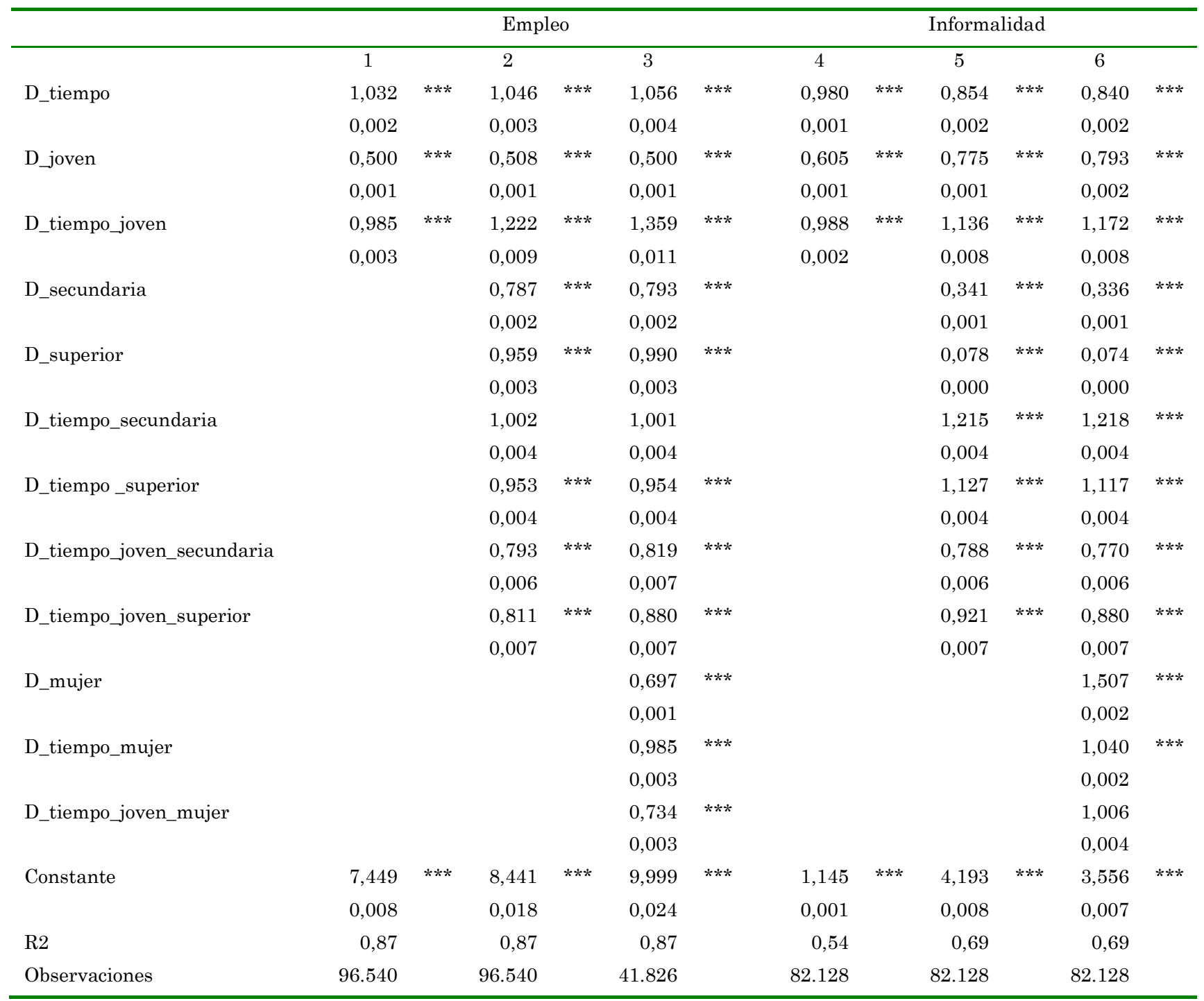

Fuente: cálculos propios con base en la GEIH.

Nota: ${ }^{* *} \mathrm{p}<0.01,{ }^{* *} \mathrm{p}<0.05,{ }^{*} \mathrm{p}<0.10$. 


\section{Cuadro 4: Resultados (odds ratios) del modelo logit (cuarto trimestre).}

\begin{tabular}{|c|c|c|c|c|c|c|c|c|c|c|c|c|}
\hline \multirow[b]{3}{*}{ D_tiempo } & \multicolumn{6}{|c|}{ Empleo } & \multicolumn{6}{|c|}{ Informalidad } \\
\hline & \multicolumn{2}{|l|}{1} & \multicolumn{2}{|l|}{2} & \multicolumn{2}{|l|}{3} & \multicolumn{2}{|l|}{4} & \multicolumn{2}{|l|}{5} & \multicolumn{2}{|l|}{6} \\
\hline & 1,105 & $* * *$ & 1,073 & $* * *$ & 1,056 & $* * *$ & 1,005 & $* * *$ & 0,907 & $* * *$ & 0,908 & $* * *$ \\
\hline & 0,002 & & 0,004 & & 0,004 & & 0,001 & & 0,002 & & 0,002 & \\
\hline \multirow[t]{2}{*}{ D_joven } & 0,492 & $* * *$ & 0,511 & $* * *$ & 0,499 & $* * *$ & 0,581 & $* * *$ & 0,734 & $* * *$ & 0,755 & $* * *$ \\
\hline & 0,001 & & 0,001 & & 0,001 & & 0,001 & & 0,001 & & 0,001 & \\
\hline \multirow[t]{2}{*}{ D_tiempo_joven } & 0,923 & $* * *$ & 0,880 & $* * *$ & 0,921 & $* * *$ & 0,938 & $* * *$ & 0,697 & $* * *$ & 0,721 & $* * *$ \\
\hline & 0,003 & & 0,007 & & 0,008 & & 0,002 & & 0,004 & & 0,005 & \\
\hline \multirow[t]{2}{*}{ D_secundaria } & & & 0,710 & $* * *$ & 0,712 & $* * *$ & & & 0,348 & $* * *$ & 0,344 & $* * *$ \\
\hline & & & 0,002 & & 0,002 & & & & 0,001 & & 0,001 & \\
\hline \multirow[t]{2}{*}{ D_superior } & & & 0,809 & $* * *$ & 0,840 & $* * *$ & & & 0,078 & $* * *$ & 0,074 & $* * *$ \\
\hline & & & 0,002 & & 0,003 & & & & 0,000 & & 0,000 & \\
\hline \multirow[t]{2}{*}{ D_tiempo_secundaria } & & & 1,043 & $* * *$ & 1,048 & $* * *$ & & & 1,180 & $* * *$ & 1,177 & $* * *$ \\
\hline & & & 0,004 & & 0,004 & & & & 0,003 & & 0,003 & \\
\hline \multirow[t]{2}{*}{ D_tiempo_superior } & & & 1,031 & $* * *$ & 1,022 & $* * *$ & & & 1,152 & $* * *$ & 1,159 & $* * *$ \\
\hline & & & 0,005 & & 0,005 & & & & 0,004 & & 0,004 & \\
\hline \multirow[t]{2}{*}{ D_tiempo_joven_secundaria } & & & 1,073 & $* * *$ & 1,110 & $* * *$ & & & 1,195 & $* * *$ & 1,171 & $* * *$ \\
\hline & & & 0,009 & & 0,009 & & & & 0,008 & & 0,008 & \\
\hline \multirow[t]{2}{*}{ D_tiempo_joven_superior } & & & 1,013 & & 1,099 & $* * *$ & & & 1,714 & $* * *$ & 1,644 & $* * *$ \\
\hline & & & 0,009 & & 0,009 & & & & 0,012 & & 0,011 & \\
\hline \multirow[t]{2}{*}{ D_mujer } & & & & & 0,650 & $* * *$ & & & & & 1,500 & $* * *$ \\
\hline & & & & & 0,001 & & & & & & 0,002 & \\
\hline \multirow[t]{2}{*}{ D_tiempo_mujer } & & & & & 1,031 & $* * *$ & & & & & 0,998 & \\
\hline & & & & & 0,003 & & & & & & 0,002 & \\
\hline \multirow[t]{2}{*}{ D_tiempo_joven_mujer } & & & & & 0,830 & $* * *$ & & & & & 0,974 & $* * *$ \\
\hline & & & & & 0,004 & & & & & & 0,004 & \\
\hline \multirow[t]{2}{*}{ Constante } & 9,594 & $* * *$ & 12,071 & $* * *$ & 14,908 & $* * *$ & 1.156 & $* * *$ & 4.129 & $* * *$ & 3,490 & $* * *$ \\
\hline & 0,011 & & 0,030 & & 0,040 & & 0.001 & & 0.007 & & 0,007 & \\
\hline $\mathrm{R} 2$ & 0,89 & & 0,89 & & 0,89 & & 0,55 & & 0,68 & & 0,69 & \\
\hline Observaciones & 99.084 & & 99.084 & & 99.084 & & 87.047 & & 87.047 & & 87.047 & \\
\hline
\end{tabular}

Fuente: cálculos propios con base en la GEIH.

Nota: ${ }^{* * *} \mathrm{p}<0.01,{ }^{* *} \mathrm{p}<0.05,{ }^{*} \mathrm{p}<0.10$. 
En el caso de la informalidad, los resultados sugieren que la probabilidad de ser informal en Colombia cayó durante el periodo analizado (columnas 4, 5 y 6 ). Este resultado positivo para la calidad de la ocupación urbana no se reflejó en general para el grupo de los jóvenes beneficiaros de la ley. El ratio de probabilidades indica que es 1,17 veces más probable ser joven informal en 2011 que en 2010 (Columna 6). Los únicos grupos que parecen beneficiarse en términos de calidad de empleo en el corto plazo son los más educados. En resumen, si bien es cierto se presentó una reducción inmediata en la probabilidad de encontrar empleo para el grupo de los jóvenes más cualificados, quienes lograron emplearse de estos grupos, lo hicieron bajo unas mejores condiciones.

Con el propósito de comparar los resultados en el cuarto trimestre de 2010 con los del 2011, en el cuadro 4 se presentan los resultados de las estimaciones del modelo logit en odds ratio. Los resultados en términos de empleo indican que el ratio de probabilidades ahora es menor. En términos de calidad de empleo, los resultados sugieren que casi un año después de la aplicación de la reforma la informalidad disminuyó para los jóvenes en general, pero aumentó para los más educados (el ratio de probabilidades indica que es 1.64 veces más probable ser profesional informal en 2011 que en 2010). En general, los resultados sobre el grupo de los más educados implican que el efecto positivo que tuvo la reforma sobre la informalidad en este grupo fue sólo temporal y se desvaneció a medida que transcurrieron los meses.

\section{Conclusiones}

La inserción exitosa de los jóvenes en los mercados de trabajo constituye un tema de investigación relevante en el plano académico y es crucial en el debate de la política económica y social de los países. El tipo de trabajo, la estabilidad y calidad de la remuneración son aspectos claves al inicio de la vida laboral que determinan trayectorias laborales. Evaluar la efectividad de las políticas públicas implementadas para tal fin es por tanto un ejercicio necesario para la continuación o rediseño de programas en el futuro.

En este documento se evaluó el efecto de la ley colombiana 1429 de 2010 denominada "ley del primer empleo" sobre la probabilidad tanto de encontrar un empleo como de tener un empleo informal para los jóvenes. El concepto de informalidad utilizado es el del DANE que establece que un trabajador es informal en Colombia si cumple alguna de las siguientes condiciones: ser un asalariado que trabaja en una empresa privada que ocupa hasta 5 personas; ser un trabajador familiar sin 
remuneración; ser un empleado doméstico o jornalero o peón o patrón o trabajador cuenta propia en una empresa hasta de cinco trabajadores.

Para evaluar el efecto de la ley sobre diferentes grupos sociodemográficos, se utilizó un modelo de diferencias en diferencias. De acuerdo con los resultados econométricos, la probabilidad de encontrar empleo en el corto plazo aumentó en general para el grupo de los jóvenes. Este efecto, sin embargo, se distribuyó de forma heterogénea entre ellos. Por niveles educativos, los jóvenes más cualificados afrontaron una menor probabilidad de encontrar trabajo. Este resultado sugeriría que el empleo generado estuvo concentrado inicialmente en sectores que utilizan mano de obra poco calificada.

En el caso de la informalidad laboral, los resultados del modelo sugieren que para los jóvenes en general, la informalidad aumentó en el corto plazo (contrario a la tendencia nacional). Sólo el grupo de jóvenes más educados parece haberse beneficiado en este caso en el periodo inmediatamente posterior a la reforma. En resumen, los resultados indican que si bien es cierto que se presentó una reducción en la probabilidad de conseguir trabajo para los más educados en el corto plazo, quienes lograron emplearse lo hicieron en mejores condiciones. Sin embargo, el efecto positivo de la ley sobre los niveles de formalización de los más educados parece ser sólo temporal, ya que a medida que trascurren los meses el efecto se desvanece.

De acuerdo con los resultados, parece conveniente replantear la efectividad de una política de generación de empleo y formalización laboral enfocada únicamente en función de incentivos tributarios y fiscales a las empresas. El alcance de la política no sólo evidencia la ausencia de una política estructural de inserción y calidad de empleo de los jóvenes en el país, sino que cuestiona el enfoque adoptado, consistente en la puesta en marcha de ciertos incentivos.

\section{Bibliografía}

Alturo, C. (2015), "Impacto tributario de la Ley 1429 del 2010 en la pequeña empresa en El Espinal, Tolima”, Ensayos de Economía, 25(46), enero-junio, 121-134.

Amarante, V.; Arim, R. y Santamaría, M. (2005), "Los efectos de la reforma laboral de 2002 en el mercado laboral colombiano", Perfil de Coyuntura Económica, 6, septiembre, 67-82. 
Bernal, R. y peña, X. (2014), Guía práctica para la evaluación de impacto, Bogotá, Universidad de los Andes, Facultad de Economía.

Card, D. (1990), "The impact of the Mariel boat lift on the Miami labor market”, Industrial and Labor Relations Review, 43(2), 245-257.

Cárdenas, M. y Barrera, F. (2003), "Análisis del impacto de la reforma laboral sobre la demanda de trabajo”, Coyuntura Social, 38-48.

Castaño, E.; López, H. y Rhenals, R. (2004), "La reforma laboral de 2002 y sus impactos: in medio stat virtus", Perfil de Coyuntura Económica, 4, diciembre, 41-69.

Congreso de Colombia (2010), Ley de Formalización y Generación de Empleo, Ley 1429 de 2010.

Departamento Administrativo Nacional de Estadística-DANE (2015), Gran Encuesta Integrada de Hogares GEIH.

Econometría y SEI S.A. (Sistemas Especializados de Información) (2013), "Evaluación a la implementación de la ley 1429 de 2010 y diseño e implementación de un sistema de seguimiento y evaluación", diciembre 2013, 1-74.

Espinel, J. (2016), "Evaluación de resultados de la ley 1429 de 2010- de formalización y generación de empleo-vista desde los beneficios otorgados en reducción de aportes parafiscales para las pequeñas empresas en la ciudad de Bogotá", Tesis para optar al título de Magister en Administración, Universidad Nacional de Colombia, Bogotá, Colombia.

Farné, S. (2012), “Acciones para la generación de empleo y mejoramiento del dialogo social en Colombia", FESCOL (Friedrich Ebert Stiftung en Colombia), noviembre, 1-18.

Farné, S. (ed.) (2011), “¿La ley 1429 de 2010 ha formalizado el empleo en Colombia?", Boletín del Observatorio del Mercado de Trabajo y La Seguridad Social, 13, diciembre, 1-44.

Gaviria, A. (2004), "Ley 789 de 2002: ¿funcionó o no?”, Documentos CEDE, 3140, noviembre, 1-48. 
Guataquí, J. y García, A. (2009), "Efectos de la reforma laboral: ¿Más trabajo y menos empleos?", Serie Documentos de Trabajo, 63, mayo, 1-47.

Isaza, J. (2003), "Flexibilización Laboral: Un análisis de sus efectos sociales para el caso colombiano", Revista Equidad y Desarrollo, 1, noviembre, 9-40.

Jaramillo, I. (2016), "Desempleo juvenil en Colombia", FESCOL (Friedrich Ebert Stiftung en Colombia), Mayo, 1-20.

López, S. (2007), "Éxitos y fracasos de la ley 789 en el área metropolitana de cali y yumbo: una evaluación teórica y empírica", Centro de Investigaciones y Documentación Socioeconomica, 98, abril, 1-37.

Narváez, A. (2013), "Efectos de la Ley 789 sobre la demanda laboral manufacturera colombiana 2001-2006”, Finanzas y Política Económica, 5(1), enero-junio, 79-94.

Núñez, J. (2005), "Éxitos y fracasos de la reforma laboral en Colombia", Documento CEDE, 43, julio, 1-34.

ILO (2016), "World employment social outlook: Trends for youth 2016", International Labour Organization, Ginebra.

Polanco, J.; Cerón, J. y Franco, D. (2015), "Impacto de la reforma laboral de 2002 en la tasa desempleo en la ciudad de Cali”, Magazín Empresarial, 11(25), diciembre, 31-38.

Rodríguez, R. (2013), "Equidad laboral para mujeres mayores de 40 años en Colombia”, Centro de Estudios Distributivos, Laborales y SocialesCEDLAS, 144, mayo, 1-37.

Wooldridge, J. (2013), Introductory Econometrics. A modern approach, $5^{\text {th }}$ Edition, South-Western, Cengage Learning. 